\title{
The Role of Embodiment in the Perception of Music
}

\author{
MARC LEMAN [1] \\ IPEM, Department of Musicology, Ghent University, Belgium \\ PIETER-JAN MAES \\ IPEM, Department of Musicology, Ghent University, Belgium
}

\begin{abstract}
Since its breakthrough at the beginning of the twenty-first century, the embodied music cognition theory has inspired empirical research on the role of actionperception couplings in musical activities. The integration of novel technologies and analysis methods inspired empirical research advancing knowledge regarding the role of embodiment in music perception and musical signification processes. In this paper, we present recent and on-going research in the field of embodied music cognition, with a focus on studies conducted at IPEM, the research laboratory in systematic musicology at Ghent University, Belgium. Attention is devoted to encoding/decoding principles underlying musical expressiveness, synchronization and entrainment, and action-based effects on music perception. The discussed empirical findings demonstrate that embodiment is only one component in an interconnected network of sensory, motor, affective, and cognitive systems involved in music perception. Currently, these findings drive the embodiment theory towards a more dynamical approach in which the interaction between various internal processes and the external environment are of central importance. Additionally, this approach envisions practical outcomes in the field of music affect research, wellbeing, healing, sports, music engineering, and brain studies.
\end{abstract}

Submitted 2014 October 20; accepted 2014 October 30.

KEYWORDS: musicology, embodied music cognition, action, perception

THE goal of the present paper is to give a review of current research conducted in the field of embodied music cognition. We thereby focus on the role of embodiment in the perception of music. Over the past decade, embodied cognition has become an influential paradigm in music research. Embodied music cognition is representative of the so-called "pragmatic turn in cognitive science" (Engel, Maye, Kurthen, \& König, 2013, p. 202), a concept that refers to the action-oriented focus that emerged in cognitive science (Prinz, Beisert, \& Herwig, 2013; Stewart, Gapenne, \& Di Paolo, 2010). The focus of embodied cognition is closely linked to related proposals such as grounded (Barsalou, 2010) and extended cognition (Clark, 2008). Embodied cognition thereby puts the emphasis on the role of the human body as a mediator for meaning formation. Its role in meaning formation can be seen as a special case of "grounded" cognition (when concepts are grounded in sensorimotor networks) or as a special case of "extended" cognition (when humans use technology to "reach out" into the musical environment). The perspective of embodied music cognition implies that the human motor system, gestures and body movements play an important role in music perception (Leman, 2007; Godøy \& Leman, 2009). The central idea is that an intentional level of musical interaction is established through corporeal articulations and imitations of sensed physical information provided by the musical environment. Empirical studies thereby focus on the role of corporeal and corporeal-technological mediators (e.g. Nijs \& Leman, 2014) and on the role of expressive gestures in music playing and music listening (e.g. Desmet, et al., 2012; Maes, Van Dyck, Lesaffre, Leman, \& Kroonenberg, 2014).

The interest in embodiment can be traced back to historical work (e.g. Becking \& Nettheim, 2011; Truslit, 1938; Hevner, 1935), in which music perception is linked with gesturing, or empathic moving along with the flow of the music, as a method for grasping musical intentions, or with affective and emotional appraisal and arousal. However, it is only recently, and thanks to novel technologies that facilitated the recording of human movement, that embodiment has become a leading research paradigm in musicology (Leman, 2007).

Therefore, it is timely to reflect on the power of the embodiment paradigm. There is a trend to broaden the perspective of embodied music cognition and to link it to musical affect research (e.g. Cochrane, Fantini, \& Scherer, 2013), wellbeing (e.g. MacDonald, Kreutz, \& Mitchell, 2012), healing (e.g. Koen, Lloyd, Barz, \& Brummel-Smith, 2011), music engineering (e.g. Kirke \& Miranda, 2013), and brain studies (e.g. Arbib, 2013), suggesting that the power of embodiment can be put in a 
broader context embracing sister-disciplines. However, there is also a trend to narrow down the perspective of embodiment, suggesting that the evidence for embodied cognition is generally weak and that more effort is needed to prove the role of embodiment in music perception. Several studies have introduced new movement analysis methods, showing that there is a strong effect of music perception on bodily behaviour (e.g. Sievers, Polansky, Casey, \& Wheatley, 2013; Toiviainen, Luck, \& Thompson, 2010; Leman \& Naveda, 2010; Naveda \& Leman, 2010). However, one could argue that the mere observation that music affects body movement, does not support the claim that music cognition is embodied. After all, it is quite evident that perception drives the motion and emotion system. There is nothing in the paradigm of music cognition to say that there is no movement involved when we perceive music (Jackendoff \& Lerdahl, 2006).

In order to highlight the role of embodiment in music perception, there are actually two possibilities. One is to show that embodiment plays a core role in an interconnected network of cognitive and emotive functions. This network is further crucial in affect processing, conceptualisation, tool use and the entire array of functions needed to make sense of music. The second is to show that embodiment is more than just an effect of music on action, and that the effect of action on music perception is essential in making sense of music (Maes, Leman, Palmer, \& Wanderley, 2014; Leman \& Maes, 2014). While the former approach broadens the perspective, the latter approach tends to narrow down the perspective in order to find empirical evidence for a specific hypothesis. However, both approaches do not exclude each other. In fact, they are complementary and necessary for the future development of embodied music cognition.

In what follows we give an overview of some of our recent studies (mostly from our laboratory, IPEM, http://www.ipem.ugent.be) that address the role of embodiment in the perception of music. We start with an overview of studies that address the encoding and decoding of musical expressiveness and entrainment. These studies tend to broaden the perspective of the nature of music perception, seen from the viewpoint of embodied cognition. Next we give an overview of studies that address the role of actions in perception. These studies tend to go deeper into specific issues that are relevant as direct proofs of the role of embodiment in music perception. We conclude with some general reflections on embodiment as a paradigm for music research.

\section{UNDERSTANDING ENCODING AND DECODING OF MUSICAL EXPRESSIVENESS IN RELATION TO EMBODIMENT}

The influence of music perception on human action is often seen as an indirect support for embodiment theory. We believe that the study of this influence is interesting, especially in view of a better understanding of the principles that underlie musical sense-giving activities. Embodiment thereby provides an interesting explanatory framework for understanding musical expression in performance and perception. Embodiment assumes the existence of mirroring processes that facilitate the encoding of expressive gestures into sounds, and the decoding of sounds into expressive gestures. Expressive gestures thereby form the common basis on which humans base their sense-giving engagement with music. Moreover, mirroring implies that the underlying mechanisms for musical meaning formation are rooted in sensorimotor principles. However, once we accept the idea of a close link between sound and gesture (and proof of evidence should be given by empirical studies, see below), we are committed to the fact that the influence of music perception on human action also works the other way around, namely, that music-driven gesturing may facilitate musical perception (Maes, Leman, et al., 2014). In short, encoding expression in sound obviously requires an action, but decoding expression from sound also involves actions, to capture the dynamic flow of music, and to facilitate the prediction of its expressive development. Therefore, from the viewpoint of embodiment, being able to engage in expressive encoding and/or decoding is considered to be a sense-giving activity regardless of the fact that a particular emotion is (re)cognised.

In that context, several empirical studies have been conducted at IPEM, which aimed at better understanding the sensorimotor basis for encoding expression in music. These studies led to several constructs of gesture types, such as elementary gestures, basic gestures, gesture repertoires/repositories, expressive-supporting gestures, sound-producing gestures, expressive-responding gestures and so on. In what follows, we briefly review these concepts in view of their possible role in music perception.

First consider the concept of elementary gesture, which we developed in the context of a study that addressed the playing of the guqin (the old Chinese zither) (Henbing \& Leman, 2007; Penttinen et al., 2006). The major goal of these studies was to understand expressive gesturing while listening to music (also called embodied listening). In this task, where listeners are asked to move along with the flow of the music, we observed that listeners have certain commonalities in their movements (Leman, Desmet, Styns, Noorden, \& Moelants, 2009). These commonalities may be due to the fact that listeners mirror certain aspects of the music, presumably expressive patterns. The guqin is of interest here because this is a fretless instrument that translates the movement of the finger on the string in a rather 
straightforward way into sound. It is possible to show that the music contains a trace of the playing gesture, and that listeners somehow mirror the body parts of the player that express these gestures. At this point, a distinction should be made between sound-producing gestures and expression-supporting gestures (Jensenius, Wanderley, Godøy, \& Leman, 2009). Interestingly, gestures of listeners don't reflect the sound-producing gestures but tend to reflect the expression-supporting gestures of the player's shoulders. The playing gestures themselves can be conceived as concatenations of elementary gestures, or movement primitives, that is, movements that go from one point to another point on the zither with zero-crossings in velocity marking the boundaries. All complex guqin gestures can thus be understood as concatenations of the elements of a gesture alphabet. Some gesture combinations of this alphabet are physically impossible, while other gesture combinations that are physically possible are not often executed. In other words, there are natural and cultural constraints in what constitutes a guqin gesture. The outcome of the study supported the idea that music perception draws upon gestures that listeners can mirror in their own expressive manner. The latter implies a commonality among listeners that is rooted in embodiment.

More recently, we focused on the foundations of expressive encoding and decoding, trying to understand these processes and develop an appropriate methodology. This was investigated in two other studies, namely, a study of expressive-supporting gestures during clarinet playing of a baroque music piece by Marin Marais (Desmet et al., 2012), and a study of expressive-responding gestures during listening to an excerpt of Brahms' First Piano Concerto (Maes, Van Dyck, et al., 2014; Amelynck, Maes, Martens, \& Leman, 2014).

The study on expressive-supporting gestures (Desmet et al., 2012) aimed to understand how gestures support intentional actions in musical performance. Intentional actions structure music according to local goals or musical target notes in a context of surrounding supporting notes. This structuring can be assumed to rely on a cognitive understanding of the musical dynamics of attraction and repelling, which Larson (2012) understands as musical forces. However, to implement these forces in sound, performers seem to rely on expressive-supporting gestures, which suggests that performers depend upon sensorimotor mechanisms to encode expression in sound (see also, e.g. Palmer, 1997; Vines, Krumhansl, Wanderley, \& Levitin, 2006).

The studies of expressive-responding gestures (Maes, Van Dyck, et al., 2014; Amelynck et al., 2014) show how listeners move in similar ways in response to music. Additionally, these studies investigate the extent to which the properties of the body movement responses to music link to the linguistic, metaphorical descriptions people use to describe the expressive qualities they perceive in the music. The results show a clear correlation between body movement features (i.e. action), and linguistic labels (i.e. perception) based on their underlying expressive qualities as defined by the Effort/Shape theory of Laban. Amelynck et al. (2014) confirm the findings of the guqin study that the expressiveresponding gestures of listeners have a high degree of commonality. The functional data analysis approach allowed us to describe the expressive-responding gestures of each listener on the basis of (i) the average movement of the entire group, and (ii) the specific movements that mark the differences among the members of the group. If the entire group were to move almost identically, then a large part of the variance in the movement could be captured by just taking the average movement of the entire group. If the members of the group were to move entirely differently from each other, then we would need a specific movement function for each individual. However, what we found was that a typical population of about 18 listeners could be described by only three specific movement functions (explaining $80 \%$ of the variance in the expressive-responding gestures among listeners). The results show that listeners' embodied responses to music are highly similar to each other, at least in populations that are homogeneous.

Finally, it is of interest to point to the role of repetitive gestures in dancing choreographies. While the expressive-responding gestures discussed above were spontaneous and not controlled by choreographies, there are indeed many embodied responses to music where gestures are controlled by dancing schemes. In that context, we investigated two spatiotemporal representational schemes for repetitive dance choreographies in response to music (e.g. Samba, Charleston), called basic gestures and spatiotemporal point clouds (Naveda \& Leman, 2009; Leman \& Naveda, 2010; Naveda \& Leman, 2010). Basic gestures are summaries of periodic gestures, characterising the average of one single period of the movement. When dancing, it is possible that body parts capture different periods related to the musical beat. For example, horizontal sway could be at two times the beat period, while arms would be at one time the beat period, and hands at half the beat period. Hence, the human body can reflect different frequencies present in the musical structure (see also Toiviainen et al., 2010). Interestingly, it is also possible to plot musical features onto these basic gestures. For example, it is possible to extract the time of the beat and plot a mark of the beat on the gesture at the time that corresponds with the beat. One way is to plot these features onto the basic gesture, while another way is to plot these features onto the entire periodic trajectory. The difference is that the basic gesture provides an average of that trajectory. When plotted on the entire trajectory, one typically obtains point-clouds with musical 
features. This can be done for different metrical periods (half beat, double beat, and so on). Accordingly, one obtains a spatiotemporal plot of the musical meter onto the gesture. Such graphs show interesting differences between, for example, Charleston and Samba dances, and especially between dance novices and dance experts.

The study of these spatiotemporal representations is of interest in relation to the hypothesis that sensorimotor synchronisation is guided by internal models that allow the anticipation of events (Maes, Leman, et al., 2014). The positions of the beat points may reflect reference points that play a prominent role in the anticipation of musical structures. The study on samba dance, for example, provided evidence that dancing may facilitate and disambiguate the perception of music. Being able to facilitate and disambiguate the perception of music is an important aspect of our understanding of the role of actions in music perception (see below).

Understanding music perception from the viewpoint of music cognition has had a focus on the anticipation of perceived structural components in music (Meyer, 1956; Huron, 2006; Honing, 2011). The idea is that anticipation is based on the ability to discern patterns (e.g. melodies, rhythms, timbres) that emerge from music through our senses. These patterns are compared with previously stored knowledge and used to generate expectations about music. The degree of match between the expected and the newly perceived pattern may then generate an outcome that is further processed by the emotional or motor system. However, what these studies on dancing show is that the anticipation may also be guided by body movements, and that this type of anticipation, due to its link with movement, is highly effective for facilitating and disambiguating perception. Moreover, as such the activity fits with the idea that decoding musical expressiveness is a sense-giving activity.

\section{UNDERSTANDING SYNCHRONISATION AND ENTRAINMENT}

Further evidence coming from our laboratory in favour of embodiment has been documented in studies that address synchronisation and entrainment (see also Repp \& Su, 2013). Starting with a tapping study, Van Noorden \& Moelants (1999) found a resonance frequency (or embodied eigen-frequency) around 2 $\mathrm{Hz}$, which can be interpreted as a reference for the perception of fast and slow tempi in music. Later, this eigen-frequency phenomenon was observed in several studies on walking, such as in Styns, Van Noorden, Moelants, \& Leman (2007). Meanwhile, strong empirical evidence has been provided for the existence of different entrainment effects, which all involve a pre-reflective adaptation of human movement to music. The core hypothesis is based on the idea that entrainment is a spatiotemporal phenomenon, not limited to timing only (Leman, 2012). Several of our empirical studies therefore focus on finding evidence for this claim.

An interesting result has been obtained in a study that provides direct evidence for a vigourentrainment effect (Leman, et al., 2013). To show this, we asked subjects to walk in synchrony with musical excerpts having the same tempo but different musical expression. The speed of walking is determined by two parameters, namely, the pace and the stride length, and the pace is fixed due to the synchronisation with the music. Therefore, the only parameter that could influence the speed is the stride length, which is an effect of the muscle strength. The study showed that some music has an activating effect in that subjects walk faster, while other music has a relaxing effect in that subjects walk slower, while metronome ticks (and much other music) have no effect. Interestingly, vigourentrainment, or the adaptation of muscle strength to music, depends on the expressive characteristics of the music. These can be defined in terms of acoustical features, showing that binary and ternary arrangements of the musical meter play an important role. This study revealed that music perception has an influence on the strength or vigour of movements.

Social-entrainment, or the adaptation of human movement to other humans while listening and producing music, has been studied in Van Noorden, De Bruyn, Van Noorden, \& Leman (2014). This study is based on an experiment with a group of more than 400 children who, in groups of four, had to continue the example of an avatar that tapped along with the beat of the music. Several tempi were tested, showing that children have a $2 \mathrm{~Hz}$ resonance in their propensity for tapping. The results have strengthened the evidence that the perception and production of the pulse is related to the characteristics of the human body (Van Noorden \& Moelants, 1999), as expressed in the tempo of spontaneous movements of young children (Provasi \& Bobin-Bègue, 2005) and the spontaneous tempo of adult walking (MacDougall \& Moore, 2005). Moreover, the analysis shows a social entrainment effect in the sense that the social group, in addition to the musical stimulus, also entrains children and provokes excitement among them so that they start to invent variation on their tapping.

\section{UNDERSTANDING EFFECTS OF ACTIONS ON MUSIC PERCEPTION}

Studies that explicitly address the effects of actions on music perception have recently been undertaken. They are highly relevant for the embodiment theory because they provide a direct proof of the role of 
embodiment in music perception. A crucial concept in our understanding of action-based effects on music perception is that of internal motor control models, more specifically, the so-called forward internal models. A forward internal model is a mechanism in the brain that enables prediction of the sensory outcome of planned or imagined actions. There is accumulating evidence that the sensory (e.g. auditory, visual, etc.) predictions made by these models influence the online processing of sensory information, such as music, coming from the external environment (Schütz-Bosbach \& Prinz, 2007; Witt, 2011; Halász \& Cunnington, 2012; Maes \& Leman, 2013). The particular influence of these predictions on the perception of music differs depending on the nature of the auditory information (when merely listening to music), or audio-visual information (when watching and listening to a music performance, for instance).

Maes, Leman, et al., (2014) provide an overview of the recent studies that consider the effects of actions on music perception. One of the effects of internal models is that perception is attenuated when the incoming sensory information matches the sensory prediction, while perception is facilitated when sensory input is incoherent with the sensory prediction. When sensory input is ambiguous in nature, body movements and the related sensory predictions enable disambiguation of incoming sensory information. Maes, Leman, et al., (2014) refer to the concepts of "selective attention", and "cue selection/identification" to explain how body movements performed to music may direct attention towards specific cues in the music. It is argued that the mechanisms described by these concepts can guide people's perception of structural features in the music (e.g. melody, beat, pitch, etc.) in a specific direction. Apart from structural properties, body movements may convey affective properties that relate to expressiveness and intentionality. Maes and Leman (2013) addressed the question of whether expressive body movements can condition children's perception of musical expressiveness. They trained children with a happy or a sad choreography in response to music that had an expressively ambiguous character. Afterwards, the children's perception of musical expressiveness in terms of valence and arousal was assessed. The results suggested that the expressive qualities of the movements they learned to associate with the music had a significant impact on how children perceived musical expressiveness.

In another study, by Naveda and Leman (2009), it was shown that Samba music has a polymetric ambiguity, whereas Samba dance patterns typically have binary tendencies. Accordingly, the authors suggest (p. 18) that "perception of samba may be movement-based in the sense that through self-movement (of the dancer in response to music) musical patterns get rhythmically disambiguated."

Other evidence is currently being accumulated via studies that address the effect of cognitive load on the expressiveness of a musical performance. Çorlu, Muller, Desmet, \& Leman, (2014) asked musicians to perform a piece of music which they knew from memory in two conditions. In one condition they just played the piece, while in the other condition they played the piece while counting circles and squares. Interestingly, in the condition where cognitive load was greater, musicians tended to shorten pause durations. Apparently, due to cognitive load they perceived the time of the pauses as extended in duration. Accordingly, it is assumed that their notion of timing over the course of a performance is guided by the spatial movements of the body while playing, whereas during pauses, they do not make spatial movements and estimate time by counting. Ongoing studies seem to confirm this initial finding. Studies on timing and cognitive load are promising because they reveal cognitive principles behind musical (inter)actions.

Of particular interest are studies that address the effect of emotions on music-driven movements. In one study Van Dyck, Maes, Hargreaves, Lesaffre, \& Leman (2013) examined the effect of two basic emotions, happiness and sadness, on dance movement. Participants were induced to feel emotional states of either happiness or sadness and then danced intuitively to an emotionally "neutral" piece of music, composed specifically for the experiment. The results revealed that when induced with happiness, participants moved faster, with more acceleration, and made more expanded and more impulsive movements than in the condition with sadness induced. A recent study (Van Dyck, Vansteenkiste, Lenoir, Lesaffre, \& Leman, 2014) investigated whether the induced emotion could be successfully decoded from movements (without music as extra stimulus). Observers watched a set of silent videos showing depersonalized avatars of dancers moving to an emotionally neutral musical stimulus after emotions of either sadness or happiness had been induced. Results revealed that observers were able to identify the emotional state of the dancers with a high degree of accuracy. In line with these two studies, it would be of interest to further investigate whether induced emotions and corresponding body movements, and the observation of people dancing in a particular way to music may have an influence on people's perception of the music itself.

\section{EMBODIMENT AND DYNAMIC COGNITION}

Based on the findings from our laboratory, we can distinguish two important principles underlying music perception and cognition that can inspire future research. First, it becomes evident that 
associations formed between sensory, motor, and affective processes are important for the perception of music. However, there is still a long way to go to fully understand the underlying mechanisms, in particular concerning the origin (innate/learned) and development (e.g. association learning, contingency, contiguity, etc.) of these associations. Second, it becomes apparent that music perception and cognition should be considered a dynamical phenomenon. Music perception and cognition encompass different systems, such as the auditory system, motor system, affective system, and cognitive system (e.g. meta-knowledge about a musical piece, autobiographic memories, etc.) situated within a specific environmental context. The disposition of each of these systems at a specific moment within the particular context determines the perception of incoming sensory information, here music. Accordingly, we advocate that music perception should be understood as a phenomenon that emerges from the dynamic interaction of many intertwined processes evolving over time. This means that changing one process will lead to changes in other processes that subsequently alter the general perception of the music. With the empirical research at IPEM, we tried to provide insights into the origin and development of dispositions of the various systems involved in music perception and cognition, and how they link to each other (cf. the concept of association). This research resulted in an extension of the embodied music cognition theory towards a dynamical theory of music cognition in which the interaction between the various sensorimotor, affective, and cognitive systems (attention, memory, meta-knowledge, etc.), and the external environment are of crucial importance.

\section{CONTRIBUTIONS TO A PARADIGM SHIFT IN SYSTEMATIC MUSICOLOGY}

We believe that the paradigm of embodied music research (which includes the theory, methodology and epistemology) has become an important research paradigm in empirical musicology. Gestures and action-perception couplings are now acknowledged to be an important part of the musical meaning formation process (Leman \& Maes, 2014; Maes, Leman, et al., 2014). In that respect our studies are in line with other studies (e.g. Phillips-Silver \& Trainor, 2005; Molnar-Szakacs \& Overy, 2006) and with current trends in cognitive research (Prinz et al., 2013; Stewart et al., 2010). In the last decade the paradigm of embodied music cognition has been made stronger in terms of methodology, theoretical underpinning, and practical outcomes.

1. Firstly, studies in embodied music research have contributed to the introduction of new methods for empirical musicology, both in recording (e.g. sensors, mocap) and analysing (e.g. multivariate, and functional data analyses). In our work, these methods have been combined with audio analysis (e.g. using a new toolbox, see Varewyck, Martens, and Leman, 2013) and subjective methodologies (e.g. questionnaires that probe flow or presence). This methodology is inter- and crossdisciplinary and has much improved compared to some 10 years ago. Meanwhile the measurement and analysis of human bodily responses to music has matured, enabling new developments such as an integration of sound and gesture in spatiotemporal representations (Leman \& Naveda, 2010). Useroriented approaches have been developed in the direction of person-centred approaches in a crossdisciplinary context of collaboration with other research groups and institutions (e.g. health care institutions) (Lesaffre, Nijs, \& Leman, 2009).

2. Secondly, the research has expanded the traditional epistemology (Elschek, 1992; Leman \& Schneider, 1997; Parncutt, 2007; Honing, 2004) in the direction of an enactive approach. For example, based on the above review from our research group alone, we believe that evidence has been provided that musical anticipation of perceived structural components in music relies on an action-based component. There are many more studies from other laboratories that support this claim (Maes, Leman, et al., 2014; Leman \& Maes, 2014). Moreover, the results provide evidence that interaction with music draws upon an interconnected network of cognition, emotion, motion, and expression (e.g. Van Dyck et al., 2013). The embodied approach also drives new developments in the field of ethnomusicology (Clayton, Dueck, \& Leante, 2013; Moran, 2013; Bonini-Baraldi, 2013).

3. Last but not least, the paradigm promotes the development of practical applications or concrete music tools, whose use in settings with users, may contribute much to the development of our theory of embodied music cognition (e.g. Maes, Amelynck, Lesaffre, Arvind, \& Leman, 2013; Maes, Amelynck, \& Leman, 2012; Maes, Leman, Kochman, Lesaffre, \& Demey, 2011; Nijs \& Leman, 2014; Moens, Noorden, \& Leman, 2010; Leman, Demey, Lesaffre, Noorden, \& Moelants, 2009 Demey, Müller, \& Leman, 2013). This approach, in which theory and practice reinforce each other, is rather new for musicology. The focus on practical applications also demonstrates the power of the paradigm of embodied music cognition. An example of such a practical application is D-Jogger (Moens et al., 2014), a tool that aligns the tempo and phase of the music with body movement in walking and running. The idea for D-Jogger emerged from our research on the relationship between music and locomotion (Styns et al., 2007). Meanwhile, this tool is used to deepen our understanding the sensorimotor principles of entrainment (Moens and Leman, 2015), as well as the effect of a music-driven coupling of locomotion and respiration on energy consumption (Bardy et al., 2015). 


\section{CONCLUSION}

In this paper we addressed the question as to why embodiment might be needed in the study of music perception. After all, it is quite evident that perception drives the motion and emotion system. Based on an overview of recent studies from our laboratory, we showed that embodiment is a necessary concept for understanding music perception. However, embodiment is not only needed as a theoretical explanatory construct for musical sense-giving activity. It is also needed as a component of our understanding of the sensorimotor basis of the encoding and decoding processes involved in musical interactions.

In this paper, some of the recent empirical work from our laboratory has been reviewed in order to illustrate in what direction this understanding of the sensorimotor basis of music interaction is currently evolving. Overall, the results provide strong evidence in favour of an intricate relationship between action and perception. The global picture that emerges from these studies is the following: when perceiving music, sensorimotor mechanisms are activated that in turn drive the perception of the music. Consequently, an interaction cycle with music is established, and this cycle can be considered as the basis for a sense-giving experience.

The concept of embodiment has contributed to the development of a research paradigm that we argue is fundamentally different from previous empirical approaches to research in music perception. While effects of music on motion and emotion were previously seen as effects of perception, we believe that this is only a part of the full story. The concept of embodiment has taught us that music perception involves an active attitude on the part of the listener, which influences perception. Given that perspective, it is likely that studies of music perception should take into account the role of embodiment. After all, sense-giving musical activities could be understood as the emergent effect of the interaction between perception and action.

\section{NOTES}

[1] Address: Sint-Pietersnieuwstraat 41, B-9000 Ghent, Belgium, tel: +32 (0)9 2644125, marc.leman@ugent.be, www.ipem.ugent.be

\section{REFERENCES}

Amelynck, D., Maes, P. J., Martens, J., \& Leman, M. (2014). Expressive body movement responses to music are coherent, consistent, and low dimensional. IEEE Transactions on Systems, Man, and Cybernetics: Part B, 44(12), 2288-2301.

Arbib, M. A. (2013). Language, music, and the brain: A mysterious relationship. Cambridge, MA: MIT Press.

Barsalou, L. W. (2010). Grounded cognition: Past, present, and future. Topics in Cognitive Science, 2(4), 716-724.

Bardy, B., Hoffmann, C., Moens, B., Leman, M. \& Dalla Bella, S. (2015). Music-induced stabilization of breathing and moving. Annals of the New York Academy of Sciences, 1337, 94-100.

Becking, G., \& Nettheim, N. (2011). How musical rhythm reveals human attitudes. Frankfurt am Main, Germany: Peter Lang Press.

Bonini-Baraldi, F. (2013). Tsiganes, musique et empathie. Paris: Editions de la Maison des sciences de l'homme.

Clark, A. (2008). Supersizing the mind: Embodiment, action, and cognitive extension. Oxford, UK: Oxford University Press.

Clayton, M., Dueck, B., \& Leante, L. (2013). Experience and meaning in music performance. Oxford, UK: Oxford University Press.

Cochrane, T., Fantini, B., \& Scherer, K. R. (Eds.). (2013). The emotional power of music: Multidisciplinary perspectives on musical arousal, expression, and social control. Oxford, UK: Oxford University Press. 
Çorlu, M., Muller, C., Desmet, F., \& Leman, M. (2014). The consequences of additional cognitive load on performing musicians. Psychology of Music, doi: 10.1177/0305735613519841.

Demey, M., Müller, C., \& Leman, M. (2013). DanSync: A platform to study entrainment and jointaction during spontaneous dance in the context of a social music game. In M. Mancas, N. d'Alessandro, X. Siebert, B. Gosselin, C. Valderrama, \& T. Dutoit (Eds.), Intelligent technologies for interactive entertainment (Vol. 124, pp. 124-135). Berlin, Germany: Springer-Verlag.

Desmet, F., Nijs, L., Demey, M., Lesaffre, M., Martens, JP., \& Leman, M. (2012). Assessing a clarinet player's performer gestures in relation to locally intended musical targets. Journal of New Music Research, 41(1), 31-48.

Elschek, O. (1992). Die Musikforschung der Gegenwart, ihre Systematik, Theorie und Entwicklung. Föhrenau, Austria: E. Stiglmayr.

Engel, A. K., Maye, A., Kurthen, M., \& König, P. (2013). Where's the action? The pragmatic turn in cognitive science. Trends in Cognitive Sciences, 17(5), 202-209.

Godøy, R. I., \& Leman, M. (2009). Musical gestures: Sound, movement, and meaning. New York, NY: Routledge.

Halász, V., \& Cunnington, R. (2012). Unconscious effects of action on perception. Brain Sciences, 2(2), 130-146.

Henbing, L., \& Leman, M. (2007). A gesture-based typology of sliding-tones in guqin music. Journal of New Music Research, 36(2), 61-82.

Hevner, K. (1935). Expression in music: A discussion of experimental studies and theories. Psychological Review, 42(2), 186-204.

Honing, H. (2004). The comeback of systematic musicology: New empiricism and the cognitive revolution. Tijdschrift voor Muziektheorie, 9(3), 241.

Honing, H. (2011). Musical cognition: A science of listening. Piscataway, NJ: Transaction Publishers.

Huron, D. B. (2006). Sweet anticipation: Music and the psychology of expectation. Cambridge, MA: MIT Press.

Jackendoff, R., \& Lerdahl, F. (2006). The capacity for music: What is it, and what's special about it? Cognition, 100(1), 33-72.

Jensenius, A. R., Wanderley, M. M., Godøy, R. I., \& Leman, M. (2009). Musical gestures: Concepts and methods in research. In R. I. Godøy \& M. Leman (Eds.), Musical gestures: Sound, movement, and meaning (pp. 12-35). New York, NY: Routledge.

Kirke, A., \& Miranda, E. R. (2013). Guide to computing for expressive music performance. Berlin, Germany: Springer-Verlag.

Koen, B., Lloyd, J., Barz, G., \& Brummel-Smith, K. (2011). The Oxford handbook of medical ethnomusicology. Oxford, UK: Oxford University Press.

Larson, S. (2012). Musical forces: Motion, metaphor, and meaning in music. Indiana, IN: Indiana University Press.

Leman, M. (2007). Embodied music cognition and mediation technology. Cambridge, MA: MIT Press.

Leman, M. (2012). Musical entrainment subsumes bodily gestures: Its definition needs a spatiotemporal dimension. Empirical Musicology Review, 7(1-2), 63-67.

Leman, M., Demey, M., Lesaffre, M., Noorden, L. van, \& Moelants, D. (2009). Concepts, technology and assessment of the social music game Sync-in-Team. In J. Calder (Ed.), Proceedings of the 2009 International Conference on Computational Science and Engineering (ICCSE) Volume 4, pp. 837-842. 
Leman, M., Desmet, F., Styns, F., Noorden, L. van, \& Moelants, D. (2009). Sharing musical expression through embodied listening: A case study based on chinese guqin music. Music Perception, 26(3), 263278.

Leman, M., \& Maes, P. J. (2014). Music perception and embodied music cognition. In L. Shapiro (Ed.), Routledge handbook of embodied cognition (pp.81-89). New York, NY: Routledge.

Leman, M., Moelants, D., Varewyck, M., Styns, F., Noorden, L. van, \& Martens, JP. (2013). Activating and relaxing music entrains the speed of beat synchronized walking. PLoS ONE, 8(7), e67932.

Leman, M., \& Naveda, L. A. (2010). Basic gestures as spatiotemporal reference frames for repetitive dance/music patterns in samba and charleston. Music Perception, 28(1), 71-91.

Leman, M., \& Schneider, A. (1997). Origin and nature of cognitive and systematic musicology: An introduction. In M. Leman (Ed.), Music, gestalt, and computing: Studies in cognitive and systematic musicology, Volume 1317 (pp. 13-29). Berlin, Germany: Springer-Verlag.

Lesaffre, M., Nijs, L., \& Leman, M. (2009). Interacting with music mediation technology for hearing impaired: First tests with normal hearing subjects. In Proceedings of the 2009 European Society for the Cognitive Sciences of Music Conference (ESCOM), pp. 267-270.

MacDonald, R., Kreutz, G., \& Mitchell, L. (2012). Music, health, and wellbeing. Oxford, UK: Oxford University Press.

MacDougall, H. G., \& Moore, S. T. (2005). Marching to the beat of the same drummer: The spontaneous tempo of human locomotion. Journal of Applied Physiology, 99(3), 1164-1173.

Maes, P.-J., Amelynck, D., \& Leman, M. (2012). Dance-the-Music: An educational platform for the modeling, recognition and audiovisual monitoring of dance steps using spatiotemporal motion templates. EURASIP Journal on Advances in Signal Processing, 35(1), 1-16.

Maes, P.-J., Amelynck, D., Lesaffre, M., Arvind, D. K., \& Leman, M. (2013). The "Conducting Master": An interactive, real-time gesture monitoring system based on spatiotemporal motion templates. International Journal of Human-Computer Interaction, 29(7), 471-487.

Maes, P.-J., \& Leman, M. (2013). The influence of body movements on children's perception of music with an ambiguous expressive character. PLOS ONE, 8(1), e54682.

Maes, P.-J., Leman, M., Kochman, K., Lesaffre, M., \& Demey, M. (2011). The “One-Person Choir”: A multidisciplinary approach to the development of an embodied human-computer interface. Computer Music Journal, 35(2), 22-35.

Maes, P.-J., Leman, M., Palmer, C., \& Wanderley, M. M. (2014). Action-based effects on music perception. Frontiers in Psychology, 4, 1-14.

Maes, P.-J., Van Dyck, E., Lesaffre, M., Leman, M., \& Kroonenberg, P. M. (2014). The coupling of action and perception in musical meaning formation. Music Perception, 32(1), 67-84.

Meyer, L. B. (1956). Emotion and meaning in music. Chicago, IL: University of Chicago Press.

Moens, B. \& Leman, M. (2015). Alignment strategies for the entrainment of music and movement rhythms. Annals of the New York Academy of Sciences, 1337, 86-93.

Moens, B., Muller, C., van Noorden, L., Franek, M., Celie, B., Boone, J., Bourgois, J., \& Leman, M. (2014). Encouraging spontaneous synchronisation with D-Jogger, an adaptive music player that aligns movement and music. PLOS ONE. http://dx.plos.org/10.1371/journal.pone.0114234

Moens, B., Noorden, L. van, \& Leman, M. (2010). D-Jogger: Syncing music with walking. In Proceedings of the Sound and Music Computing Conference (SMC), pp. 451-456. 
Molnar-Szakacs, I., \& Overy, K. (2006). Music and mirror neurons: From motion to emotion. Social Cognitive and Affective Neuroscience, 1(3), 235-241.

Moran, N. (2013). Music, bodies and relationships: An ethnographic contribution to embodied cognition studies. Psychology of Music, 41(1), 5-17.

Naveda, L. A., \& Leman, M. (2009). A cross-modal heuristic for periodic pattern analysis of Samba music and dance. Journal of New Music Research, 38(3), 255-283.

Naveda, L. A., \& Leman, M. (2010). The spatiotemporal representation of dance and music gestures using topological gesture analysis (TGA). Music Perception, 28(1), 93-111.

Nijs, L., \& Leman, M. (2014). Interactive technologies in the instrumental music classroom: A longitudinal study with the Music Paint Machine. Computers \& Education, 73, 40-59.

Palmer, C. (1997). Music performance. Annual Review of Psychology, 48(1), 115-138.

Parncutt, R. (2007). Systematic musicology and the history and future of western musical scholarship. Journal of Interdisciplinary Music Studies, 1(1), 1-32.

Penttinen, H., Pakarinen, J., Välimäki, V., Laurson, M., Henbing, L., \& Leman, M. (2006). Modelbased sound synthesis of the guqin. Journal of the Acoustical Society of America, 120(6), 4052-4063.

Prinz, W., Beisert, M., \& Herwig, A. (2013). Action science: Foundations of an emerging discipline. Cambridge, MA: MIT Press.

Provasi, J., \& Bobin-Bègue, A. (2005). Tempo discrimination in 3- and 4-year-old children: Performances and threshold. Current Psychology Letters, 16(2).

Repp, B. H., \& Su, Y. H. (2013). Sensorimotor synchronization: A review of recent research (20062012). Psychonomic Bulletin \& Review, 20(3), 403-452.

Schütz-Bosbach, S., \& Prinz, W. (2007). Perceptual resonance: Action-induced modulation of perception. Trends in Cognitive Sciences, 11(8), 349-355.

Sievers, B., Polansky, L., Casey, M., \& Wheatley, T. (2013). Music and movement share a dynamic structure that supports universal expressions of emotion. Proceedings of the National Academy of Sciences of the United States of America (PNAS), 110(1), 70-75.

Stewart, J., Gapenne, O., \& Di Paolo, E. A. (2010). Enaction: Toward a new paradigm for cognitive science. Cambridge, UK: Cambridge University Press.

Styns, F., Van Noorden, L., Moelants, D., \& Leman, M. (2007). Walking on music. Human Movement Science, 26(5), 769-785.

Toiviainen, P., Luck, G., \& Thompson, M. R. (2010). Embodied meter: Hierarchical eigenmodes in music-induced movement. Music Perception, 28(1), 59-70.

Truslit, A. (1938). Gestaltung und bewegung in der musik. Berlin-Lichtenfelde, Germany: Christian Fiedrich Vieweg.

Van Dyck, E., Maes, P. J., Hargreaves, J., Lesaffre, M., \& Leman, M. (2013). Expressing induced emotions through free dance movement. Journal of Nonverbal Behavior, 37(3), 175-190.

Van Dyck, E., Vansteenkiste, P., Lenoir, M., Lesaffre, M., \& Leman, M. (2014). Recognizing induced emotions of happiness and sadness from dance movement. PLoS ONE, 9(2), e89773.

Van Noorden, L., De Bruyn, L., Van Noorden, R., \& Leman, M. (2014). Resonance and social entrainment in the synchronous reproduction of musical pulse: Developments in childhood. (in review).

Van Noorden, L., \& Moelants, D. (1999). Resonance in the perception of musical pulse. Journal of New Music Research, 28(1), 43-66. 
Varewyck, M., Martens, JP., \& Leman, M. (2013). Musical meter classification with beat synchronous acoustic features, DFT-based metrical features and support vector machines. Journal of New Music Research, 42(3), 267-282.

Vines, B. W., Krumhansl, C. L., Wanderley, M. M., \& Levitin, D. J. (2006). Cross-modal interactions in the perception of musical performance. Cognition, 101(1), 80-113.

Witt, J. K. (2011). Action's effect on perception. Current Directions in Psychological Science, 20(3), 201-206. 